\title{
Cycles and Paths in Semicomplete Multipartite Digraphs, Theorems and Algorithms: A Survey
}

\author{
G. Gutin *
}

March 9, 2003

\begin{abstract}
A digraph obtained by replacing each edge of a complete $m$-partite graph by an arc or a pair of mutually opposite arcs with the same end vertices is called a semicomplete $m$-partite digraph. We describe results (theorems and algorithms) on directed walks in semicomplete $m$ - partite digraphs including some recent results concerning tournaments.
\end{abstract}

\section{Introduction}

A digraph obtained by replacing each edge of a (simple) graph $G$ by an arc (by an arc or a pair of mutually opposite arcs) with the same end vertices is called an orientation (biorientation, respectively) of $G$. Therefore, orientations of graphs have no opposite arcs, and biorientations may have. The investigation of paths and cycles in tournaments, orientations of complete graphs, was initiated by Redei's Theorem [63] derived in 1934: each tournament contains a Hamiltonian path. In 1959, P. Camion [25] obtained necessary and sufficient conditions for the existence of a Hamiltonian cycle in a tournament. He proved that every strongly connected tournament has a Hamiltonian cycle. There are several survey articles [20, 21, 47] (the second one contains results on general digraphs too) and a book by J. Moon [58] where the properties of tournaments are considered.

J. Moon [58] and J. A. Bondy [22] were the first to consider cycles in the entire class of multipartite tournaments (orientations of complete multipartite graphs). Since the 80s, mathematicians began studying extensively cycles and paths in bipartite tournaments. The first results were described in the survey by L. W. Beineke [18]. In this period a number of results on the cycle and path structure of $m$-partite tournaments for $m \geq 3$ were obtained. A survey describing these results as well as recent results on cycles and paths in bipartite

*Department of Mathematics, Raymond and Beverly Sackler Faculty of Exact Sciences, Tel Aviv University, Ramat-Aviv, Tel-Aviv 69978, Israel 
tournaments is absent and seems to be needed. The aim of the present article is to fill in this gap and also to describe some theorems and algorithms on paths and cycles in tournaments which have been obtained recently.

Note that part of the results given in the paper are formulated not for orientations of complete multipartite graphs (usually called multipartite tournaments) but for biorientations of them (called semicomplete multipartite digraphs). In particular, we give some theorems for semicomplete digraphs (biorientations of complete graphs) instead of the more restricted class of tournaments. The motivation for considering semicomplete multipartite digraphs rather than multipartite tournaments is the following. From a theoretical point of view there is no good reason to restrict investigation to digraphs having no opposite arcs when more general results may be available. Digraphs with opposite arcs are sometimes used in order to obtain results for digraphs without opposite arcs (see [34, 72]). Moreover, total exclusion of opposite arcs from the consideration does not allow to study adequately some practical digraph models (models in social choice theory, interconnections networks, etc. [73]). That is why there are numerous papers where properties of semicomplete digraphs, semicomplete bipartite and $m$-partite $(m \geq 3)$ digraphs (see, for example, $[16,17,30,35,40,42,73])$ were investigated.

We hope that this survey will be as successful as [18] in stimulating further research on the subject.

\section{Notation and Terminology}

We consider finite directed graphs without loops and multiple arcs [23]. A digraph $D$ on $m$ disjoint vertex classes (called partite sets) is called a semicomplete m-partite or multipartite digraph (abbreviated to SMD, and for $m=2$ - to SBD) if for any two vertices $u, v$ in different partite sets either $(u, v)$ or $(v, u)$ (or both) is an arc of $D$ and there are no arcs between vertices which belong to the same partite set. Such a digraph $D$ is called ordinary if for any pair $X, Y$ of its partite sets, the set of arcs with both end vertices in $X \cup Y$ coincides with $X \times Y=\{(x, y): x \in Y, y \in Y\}$ or $Y \times X$ or $(X \times Y) \cup(Y \times X)$. A semicomplete digraph is a SMD, each part of which consists of a single vertex. A semicomplete $m$-partite digraph is called an m-partite or multipartite tournament (abbreviated to MT, and for $m=2$ - to BT) if it has no mutually opposite arcs. A tournament is a MT, each part of which consists of a single vertex.

By a cycle (path) we mean a simple directed cycle (path, respectively). An $m$-cycle ( $m$-path) is a cycle (path) which has $m$ arcs. A $(x, y)$-path is a path from $x$ to $y(x, y$ are vertices). A cycle (path) of a digraph $D$ is called Hamiltonian if it includes all the vertices of $D$. A digraph is called Hamiltonian if it contains a Hamiltonian cycle. A digraph $H$ is pancyclic if it contains a cycle of length $i$ (i-cycle) for any $3 \leq i \leq n$, where $n$ is the order of $H$; $H$ is vertex (arc) pancyclic if it has an $i$-cycle containing $x$ for any vertex (arc) $x$ of $H$, $3 \leq i \leq n$. Even pancyclicity, vertex even pancyclicity and arc even pancyclicity are defined analogously: in this case we only require cycles of all lengths $i \equiv 0 \quad(\bmod 2)$. 
A digraph $D$ is said to be strongly connected or just strong if for every pair $x, y$ vertices of $D$ there is a $(x, y)$-path and a $(y, x)$-path. A strong component of $D$ is a maximal induced subgraph of $D$. A digraph $D$ is called $k$-strongly connected $(k \geq 1)$ if for any set $X$ of at most $k-1$ vertices of $D$ the subgraph obtained by removing $X$ from $D$ is strongly connected. We call a digraph $D k$-vertex ( $k$-arc, respectively) cyclic if it contains a cycle through any subset of $k$ vertices (any set of $k$ independent arcs, respectively) of $D$.

The sets $V(D), A(D)$ are the sets of vertices and arcs of a digraph $D$. Let $k$ be an integer. A digraph $D$ is called $k$-diregular (or, simply, diregular) if $d^{+}(x)=d^{-}(x)=k$ for any $x \in V(D)$. A digraph $D$ is called almost $k$-diregular (or, simply, almost diregular) if there exist vertices $x, y$ (possibly $x=y$ ) such that $d^{+}(x)=d^{-}(y)=k-1$, and $d^{+}(z)=k$ for $z \in V(D) \backslash x, d^{-}(v)=k$ for $v \in V(D) \backslash y$. It is easy to see that a 1-diregular digraph $F$ represents a collection of vertex disjoint cycles $C_{1}, C_{2}, \ldots, C_{t}(t \geq 1)$, i.e. $F=C_{1} \cup C_{2} \cup \cdots \cup C_{t}$. Similarly, an almost 1-diregular digraph $S=C_{0} \cup C_{1} \cup C_{2} \cup \cdots \cup C_{q}$, where $C_{0}$ is a path, (which may have only one vertex), $C_{1}, C_{2}, \ldots, C_{q}$ are cycles, $V\left(C_{i}\right) \cap V\left(C_{j}\right)=\emptyset$ for $0 \leq$ $i \neq j \leq q, q \geq 0$. Let $D$ be a digraph. A 1-diregular spanning subgraph of $D$ is called a 1-difactor.

The eccentricity of the vertex $v$ (denoted by $\operatorname{ecc}(v)$ ) is the maximum of the lengths of the shortest paths from $v$ to $u$, where the maximum is taken over all vertices $u$ of $D$. The radius (diameter, respectively) of $D$ is $\min \operatorname{ecc}(v)$ ( $\max \operatorname{ecc}(v)$, respectively), where $v$ ranges over all vertices of $D$. A vertex $w$ is called a center of $D$ if $\operatorname{ecc}(w)$ is equal to the radius of $D$.

Let $D$ be a digraph, and let $x$ be a vertex of $D$, then

$$
\Gamma^{+}(x)=\{y \in V(D):(x, y) \in A(D)\}, \quad \Gamma^{-}(x)=\{z \in V(D):(z, x) \in A(D)\} .
$$

The underlying graph of a digraph $D$ is the graph obtained from $D$ by disregarding the orientations of all arcs of $D$. A digraph $D$ is called an arc-local tournament digraph [10] if for each edge $x y$ of its underlying graph every vertex of $\Gamma^{+}(x)$ is adjacent to every vertex of $\Gamma^{+}(y)$ and $\Gamma^{-}(x)$ is adjacent to every vertex of $\Gamma^{-}(y)$. It is easy to see that arc-local tournament digraphs are common generalization of bipartite tournaments and tournaments.

Let $B=B\left(r_{1}, r_{2}, r_{3}, r_{4}\right)$ be the following BT, which will be useful several times later. Let $R_{1}, \ldots, R_{4}$ be pairwise disjoint independent sets of vertices, $\left|R_{i}\right|=r_{i}(i=1,2,3,4)$. Define $V(B)=R_{1} \cup \cdots \cup R_{4}$ and let $A(B)$ consist of all the arcs from $R_{i}$ to $R_{i+2}, i=1,2$, from $R_{3}$ to $R_{2}$ and from $R_{4}$ to $R_{1}$.

For a real number $a$, denote by $[a]$, as usual, the integer part of a. Throughout this paper, $n$ denotes the number of vertices of the digraph or graph considered.

\section{Paths in SMDs}

The following result is proved in [40].

Theorem 3.1 Let $D$ be a SMD. Then 
1) for any almost 1-diregular subgraph $F$ of $D$ there is a path $P$ of $D$ satisfying $V(P)=$ $V(F)$ (if $F$ is a maximum almost 1-diregular subgraph each such path is a longest path of $D)$;

2) there exists an $O\left(n^{3}\right)$ algorithm for finding a longest path in $D$.

The first half of Theorem 3.1 follows from the following:

Lemma 3.2 Let $D$ be a $S M D$ and $P, C$ a path and a cycle having no common vertices; then the subgraph of $D$ induced by $V(P) \cup V(C)$ contains a Hamiltonian path.

In order to describe the algorithm mentioned in Theorem 3.1 we first consider a construction by N. Alon (cf. [40]) which allows one to find efficiently a 1-diregular subgraph with maximum order of a given digraph $D$. Let $B=B(D)$ be a bipartite weighted graph, such that $\left(X, X^{\prime}\right)$ is the partition of $B$, where $X=V(D), X^{\prime}=\left\{x^{\prime}: x \in X\right\} ; x y^{\prime} \in E(B)$, if and only if either $(x, y) \in A(D)$ or $x=y$. The weight of an edge $x y^{\prime}$ of $B$ equals 1 if $x \neq y$ and equals 2 , otherwise. It is easy to see that solving the assignment problem for $B$ (in time $O\left(n^{3}\right)$, cf. [61] ) and, then, removing all the edges with weight 2 from the solution, we obtain a set of edges of $B$ corresponding to some 1-diregular subgraph $F$ of $D$ of maximum order.

For a cycle $C$ and a vertex $x$ on it, denote by $C_{x}$ the path obtained from $C$ by deleting the arc ending at $x$.

Now we are ready to describe the algorithm.

Algorithm 3.3 for finding a longest path in a SMD.

Input. A semicomplete multipartite digraph $D$.

Output. A longest path $H$ of $D$.

Step 1. Construct the digraph $D^{\prime}$ with

$$
V\left(D^{\prime}\right)=\{x\} \cup V(D)(x \notin V(D)), A\left(D^{\prime}\right)=A(D) \cup\{(x, y),(y, x): y \in V(D)\}
$$

Find a 1-diregular subgraph $F^{\prime}$ of $D^{\prime}$ of maximum order. Let $C_{0}, C_{1}, \ldots, C_{t}(t \geq 0)$ be the cycles of $F^{\prime}$, and suppose $x \in V\left(C_{0}\right)$ (it is easy to see that $x \in F^{\prime}$ ). Find $P=C_{0}-x$, and put

$$
F:=P \cup C_{1} \cup \cdots \cup C_{t} .
$$

Note that $F$ is almost a 1-diregular subgraph of $D$ of maximum order. We shall construct a path on all the vertices of $F$ - this will clearly be a longest path.

Step 2. If $t=0$, then $H:=P$, and we have finished. Otherwise put $C:=C_{t}, t:=t-1$. Let

$$
P=\left(x_{1}, x_{2}, \ldots, x_{m}\right), C=\left(y_{1}, y_{2}, \ldots, y_{k}, y_{1}\right) .
$$


Step 3. If $\Gamma^{-}\left(x_{1}\right) \cap V(C) \neq \emptyset$, then pick any $z \in \Gamma^{-}\left(x_{1}\right) \cap V(C)$, put $P:=\left(C_{z^{+}}, P\right)$, where $z^{+}$is the vertex following $z$ in $C$, and go back to Step 2. Analogously, if there exists $y \in \Gamma^{+}\left(x_{m}\right) \cap V(C)$ put $P:=\left(P, C_{y}\right)$, and go back to Step 2 .

Step 4. For $i=1,2, \ldots, m-1 ; j=1,2, \ldots, k$ if $\left(y_{j}, x_{i+1}\right),\left(x_{i}, y_{j+1}\right) \in A(D)$, then let $P$ be the path containing the fragment of $P$ from $x_{1}$ to $x_{i}$, the path $C_{y_{j+1}}$, and the fragment of $P$ from $x_{i+1}$ to $x_{m}$. Go to Step 2. If none of Steps 2,3,4 can be applied, we go to Step 5 below.

Step 5. For $j=1,2, \ldots, k ; i=1,2, \ldots, m-1$ if $i$ is minimal such that there exists $j=j(i)$ for which

$$
\left(y_{j}, x_{i+1}\right),\left(y_{j+1}, x_{i}\right) \in A(D)
$$

then let $P$ be the path containing the fragment of $P$ from $x_{1}$ to $x_{i-1}$, the vertices $y_{j+1}, x_{i}$, the fragment of $C$ from $y_{j+2}$ to $y_{j}$, and the fragment of $P$ from $x_{i+1}$ to $x_{m}$ in the given order (the direct proof of Lemma 3.2 [40] consists of showing the existence of the above mentioned $i, j=j(i)$ as well as the $\left.\operatorname{arcs}\left(x_{i-1}, y_{j+1}\right),\left(x_{i}, y_{j+2}\right)\right)$. Go to Step 2.

Lemma 3.2 can also be proved as a rather simple consequence of a sufficient condition for a SMD to be Hamiltonian, shown in [11] (see Theorem 4.8 bellow). This proof of Lemma 3.2 provides a more complicated algorithm than Algorithm 3.3.

Step 1 of Algorithm 3.3 can be executed in time $O\left(n^{3}\right)$. All the other steps can be performed in time $O\left(n^{2}\right)$. Using the maximum matching algorithm for bipartite graphs [3], one can test whether a digraph $D$ contains a 1-diregular spanning subgraph $F^{\prime}$ and find some $F^{\prime}$ in case one exists in time $O\left(n^{2.5} / \sqrt{\log n}\right)$. This implies

Corollary 3.4 1) A SMD D has a Hamiltonian path, if and only if it has an almost 1diregular spanning subgraph. 2) Testing whether D has a Hamiltonian path (and finding one of them in case it exists) can be done in time $O\left(n^{2.5} / \sqrt{\log n}\right)$.

Corollary 3.4 was derived in [35] as a generalization of the same theorem obtained for semicomplete bipartite digraphs in [33]. Using a different approach, R. Häggkvist and Y. Manoussakis gave in [46] analogous characterization of bipartite tournaments having a Hamiltonian path. Y. Manoussakis and Z. Tuza constructed in [55] an $O\left(n^{2.5} / \sqrt{\log n}\right)$ algorithm for finding a Hamiltonian path in a bipartite tournament $B$ (if $B$ has a Hamiltonian path).

Corollary 3.4 implies that any almost diregular or diregular multipartite tournament has a Hamiltonian path. This last result was also proved in [80] as a corollary of Theorem 4.13 (see below).

Recently J. Bang-Jensen [10] proved that Corollary 3.4 is also valid for arc-local tournament digraphs.

The problem of deciding whether a tournament with two given vertices $x$ and $y$, contains a Hamiltonian path with endvertices $x, y$ (the order not specified) was solved by C. Thomassen [71]. It follows from his characterization that the existence of such a path for specified vertices 
Figure 1: The exceptional tournaments where the edge between $x$ and $y$ can be oriented arbitrarily

$x, y$ can be checked in polynomial time. Considering ordinary MTs, J. Bang-Jensen, G. Gutin and J. Huang [12] obtained a generalizations of the above characterization. They proved the following:

Theorem 3.5 Let $D$ be an ordinary multipartite tournament and let $x, y$ be distinct vertices of $D$. Then $D$ has a Hamiltonian path connecting $x$ and $y$ (from $x$ to $y$ or from $y$ to $x$ ), if and only if $D$ has a path $P$ connecting $x$ and $y$ such that $D-P$ has a 1-difactor and $D$ does not satisfy any of the conditions (1) - (4) below.

(1) D is not strong and either the initial or the terminal strong component of D (or both) contains none of $x$ and $y$;

(2) $D$ is strong, $D-x$ is not strong and either $y$ belongs to neither the initial nor the terminal strong component of $D-x$, or $y$ belongs to the initial (terminal) strong component of $D-x$ and there is no path $P^{\prime}$ from $y$ to $x$ (path from $x$ to $y$, respectively) such that $D-P^{\prime}$ has a 1-difactor.

(3) $D$ is strong, $D-y$ is not strong and either $x$ belongs to neither the initial nor the terminal strong component of $D-y$, or $x$ belongs to the initial (terminal) strong component of $D-y$ and there is no path $P^{\prime}$ from $x$ to $y$ (path from $y$ to $x$, respectively) such that $D-P^{\prime}$ has a 1-difactor.

(4) $D, D-x$, and $D-y$ are all strong and $D$ is isomorphic to one of the tournaments shown in Figure 1.

An analogous characterization of all bipartite tournaments that have a Hamiltonian path between two prescribed vertices $x, y$ was derived by J. Bang-Jensen and Y. Manoussakis in 
[14]. The only difference between these two characterizations is in Condition 4: in BangJensen's and Manoussakis' theorem the set of forbidden digraphs is absolutely different from that of Theorem 3.5 and moreover infinite (see [14]). Both characterizations imply polynomial algorithms to decide the existence of a Hamiltonian path connecting two given vertices and find one (if it exists). In [71] C. Thomassen considered not only the problem of deciding if for a pair $x, y$ of vertices there is a Hamiltonian path either from $x$ to $y$ or from $y$ to $x$ but also the stronger problem of deciding if there exists a Hamiltonian $(x, y)$-path. He proved that for every pair $x, y$ of vertices of a 4-strongly connected tournament there is a Hamiltonian path starting at $x$ and ending at $y$. In $[12,14]$, the following conjecture was formulated.

Conjecture 3.6 Let $D$ be a 4-strongly connected ordinary MT (or bipartite tournament). The digraph $D$ has a Hamiltonian path from $x$ to $y$ for any pair of vertices $x, y$ of $D$ if and only if $D$ contains an $(x, y)$-path $P$ such that $D-P$ has a factor.

The radius and diameter are important invariants of a digraph. H. Landau [51] observed that the radius of any tournament is at most two and each vertex of maximum outdegree in it is a center. Obviously, any MT containing at least two vertices of indegree zero has an infinite radius. However, in case there are no such two vertices, the radius can be bounded, as shown in the following statement, proved in [34] and, independently, in [62].

Theorem 3.7 Any MT with at most one vertex of indegree zero has radius $r \leq 4$.

B. Sands, N. Sauer and R. Woodrow [65] studied monochromatic paths in arc-coloured digraphs. In particular, they proved that every tournament whose arcs are coloured with two colours contains a vertex $v$ such that for every other vertex $w$ there exists a monochromatic $(v, w)$-path. They also showed the following:

Theorem 3.8 Let $T$ be a tournament whose arcs are coloured with three colours, and whose vertices can be partitioned into disjoint blocks such that

(i) two vertices in different blocks are always connected by a red arc;

(ii) two vertices in the same block are always connected by a blue or a green arc.

Then there is a vertex $v$ of $T$ such that for every other vertex $x$ of $T$ there is a monochromatic path from $v$ to $x$.

It is easy to see that the last theorem follows from Theorem 3.7 and the first mentioned result of B. Sands, N. Sauer and R. Woodrow.

It is easy to check that Theorem 3.7 holds for the entire class of SMD. V. Petrovic and C. Thomassen [62] pointed out that Theorem 3.7 can be extended to a larger class of oriented graphs (at the cost of modifying the constant 4 ). 
Theorem 3.9 Let $G$ be a graph whose complement is a disjoint union of complete graphs, cycles and paths. Then every orientation of $G$ with at most one vertex of indegree zero has radius at most 6 .

Unlike tournaments, a vertex of maximum outdegree in a MT is not necessarily a center as proved in [27]:

Theorem 3.10 Let $T$ be a strongly connected 3-partite tournament of order $n \geq 8$. If $v$ is a vertex of maximum outdegree in $T$, then $\operatorname{ecc}(v)$ is at most $[n / 2]$ and this bound is best possible.

In the case of bipartite tournaments, it is possible to obtain more detailed results. In [37] characterizations of vertices with eccentricity $1,2,3$ or 4 were derived. Using these characterizations all bipartite tournaments with radius 1, 2, 3 or 4 were characterized.

It is easy to see [41], that if a graph $G$ has an orientation with a finite diameter (i. e., if $G$ has no bridges [64]), then the maximum diameter of such an orientation is equal to the length of the longest path in $G$. The problem of finding the minimum possible diameter of such an orientation is significantly more complicated. Denote by $f\left(m_{1}, m_{2}, \ldots, m_{k}\right)$ the minimum possible diameter of a $k$-partite tournament with partite sets of sizes $m_{1}, m_{2}, \ldots, m_{k}$. L. Soltes [66] obtained the following result.

Theorem 3.11 If $m_{1} \geq m_{2} \geq 2$, then $f\left(m_{1}, m_{2}\right)=3$ for $m_{1} \leq\left(\begin{array}{c}m_{2} \\ {\left[m_{2} / 2\right]}\end{array}\right)$, and otherwise $f\left(m_{1}, m_{2}\right)=4$.

A shorter proof of this result using the well known theorem of Sperner (cf. [1]) is given in [37]. In [41], the following result dealing with $k \geq 3$ was proved.

Theorem 3.12 If $k \geq 3$, then

1. $f\left(m_{1}, m_{2}, \ldots, m_{k}\right) \leq 3$ for all positive integers $m_{1}, m_{2}, \ldots, m_{k}$,

2. for any positive integer $m f(\underbrace{m, m, \ldots, m}_{k \text { times }})=2$ except the case $m=1, k=4$ where $f(1,1,1,1)=3$.

It is natural to pose the following:

Problem 3.13 Determine all $k$ tuples of integers $m_{1}, \ldots, m_{k}$ such that $f\left(m_{1}, \ldots, m_{k}\right)=2$. 


\section{Cycles in semicomplete multipartite digraphs}

J. A. Bondy [22] extended the above mentioned Moser's Theorem on $k$-partite $(k \geq 3)$ tournaments in the following form (this result was obtained independently in [28] as well).

Theorem 4.1 Any strongly connected $k$-partite $(k \geq 3)$ tournament contains $m$-cycles for all $m \in\{3,4, \ldots, k\}$.

\section{J. A. Bondy also showed}

Theorem 4.2 If a strongly connected $k$-partite $(k \geq 5)$ tournament has in each partite set at least two vertices, then it has a $m$-cycle with $m>k$.

In connection with the last statement he asked [22] if the inequality $m>k$ may be replaced by the equality $m=k+1$. A negative answer to this question was obtained in [30](for details see [32]). The same counter-example (as in [30,32]) was found independently by R. Balakrishnan and P. Paulraja [8]. Consider the $k$-partite $(k \geq 3)$ tournament $D_{k}$ with the partite sets $\left\{x_{i}^{(1)}, x_{i}^{(2)}\right\}, 1 \leq i \leq k$ and the arc set

$$
\begin{array}{r}
\bigcup_{j=1}^{2}\left(\left\{\left(x_{i}^{(j)}, x_{i+1}^{(j)}\right): 1 \leq i \leq k-1\right\} \cup\left\{\left(x_{m}^{(j)}, x_{i}^{(j)}\right): 3 \leq i+2 \leq m \leq k\right\}\right) \cup \\
\left\{\left(x_{i}^{(1)}, x_{m}^{(2)}\right): 1 \leq i \neq m \leq k\right\} \cup\left\{\left(x_{k}^{(2)}, x_{1}^{(1)}\right)\right\} \backslash\left\{\left(x_{1}^{(1)}, x_{k}^{(2)}\right)\right\} .
\end{array}
$$

It is easy to see that $D_{k}(k \geq 3)$ has no $(k+1)$-cycle.

In [32] it was also proved that the inequality $m>k$ (in Theorem 4.2 ) may be replaced by the inequality $k+1 \leq m \leq k+2$.

In connection with Theorem 4.1 J. A. Bondy [22] raised the question if some form of the corresponding generalization of Moon's Theorem [58] is also true. He further gave an example showing that the last generalization is not true in general.

In [28], [39] and [29] the following three restricted generalizations of Moon's theorem were obtained.

Theorem 4.3 [28] Every vertex of a strongly connected $k$-partite tournament $(k \geq 3)$ lies on a cycle that contains vertices from exactly $m$ partite sets, for all $3 \leq m \leq k$.

Theorem 4.4 [39] Let $D$ be a strongly connected $k$-partite ( $k \geq 3)$ tournament, one of the partite sets of which consists of a single vertex, say $v$. Then, for each $m \in\{3,4,, \ldots, k\}$, there is an $m$-cycle of $D$ containing $v$.

Theorem 4.5 [29] Let $D$ be a strongly connected $k$-partite $(k \geq 3)$ tournament with partite sets $V_{1}, \ldots, V_{k}$. Then, for every $m \in\{3,4,, \ldots, k\}$ and every $i \in\{1, \ldots, k\}$, there is an $m$-cycle of $D$ containing a vertex from $V_{i}$. 
Note that the last theorem implies the previous one.

W. Goddard, G. Kubicki, O. Oellermann and S. Tian [27] proved that every vertex of a strongly connected $k$-partite tournament $T(k \geq 3)$ belongs to a 3-cycle or a 4-cycle of $T$. Moreover, they obtained the following:

Theorem 4.6 Let $T$ be a k-partite tournament, $k \geq 3$. 1) If $T$ has a cycle of length $m \geq 4$ containing vertices from at least three distinct partite sets, then $T$ contains a cycle of length $m-1, m-2$ or $m-3$. 2) If $T$ has a cycle of length $m \geq 5$ containing vertices from at least three distinct partite sets, then $T$ contains a cycle of length $m-2$ or $m-3$.

The second part of Theorem 4.6 is best possible in the following sense [27]. For every $m=3 s$, for some integer $s \geq 1$, there exists a MT having cycles of lengths $m$ and $m-3$, but no cycles of length $m-2$. Further, for each odd integer $m \geq 9$, there exists a MT having cycles of lengths $m$ and $m-2$, but no cycles of length $m-3$.

In [55] and [17], the problem of the existence of a cycle containing prescribed vertices in MTs is studied. In [17], the following result is shown.

Theorem 4.7 There exist a polynomial algorithm for deciding if two vertices in a MT are on a common cycle.

J. Bang-Jensen, G. Gutin and J. Huang [11] study the Hamiltonian cycle problem for SMDs. To describe the main result of [11], we need the following definitions. Let $C$ and $Z$ be two disjoint cycles in a digraph $D$. A vertex $x \in C$ is called out-singular (in-singular)) with respect to $Z$ if $\Gamma^{-}(x) \cap V(Z)\left(\Gamma^{+}(x) \cap V(Z)\right.$, respectively) is empty. A vertex is singular if it is out-singular or in-singular. A 1-difactor $F=C_{1} \cup \cdots \cup C_{t}$ in a digraph $D$ is called good if there is no pair $C_{i}, C_{j}(1 \leq i \neq j \leq t)$ such that $C_{i}$ has singular vertices with respect to $C_{j}$ and they are all out-singular, and $C_{j}$ contains singular vertices with respect to $C_{i}$ and they are all in-singular. The main result of [11] is the following sufficient condition for a SMD to be Hamiltonian.

Theorem 4.8 Let $D$ be a SMD having a good 1-difactor $F$. Then $D$ contains a Hamiltonian cycle and one can be found in time $O\left(n^{2}\right)$ given $F$.

The following lemma is used in the proof of Theorem 4.8 in [11]. It is useful in other proofs as well (see Theorems 5.5, 5.6).

Lemma 4.9 Let $F=C_{1} \cup C_{2} \cup \cdots \cup C_{t}$ be a 1-diregular subgraph of maximum cardinality of a strongly connected $S M D D$, where $C_{i}$ is a cycle in $D(1 \leq i \leq t)$. Let, also, $F$ satisfy the following condition: for every pair $1 \leq i<j \leq t$ all arcs between $C_{i}$ and $C_{j}$ are oriented either from $C_{i}$ to $C_{j}$ or from $C_{j}$ to $C_{i}$. Then $D$ has a (longest) cycle of length $|V(F)|$ and one can find such a cycle in time $O\left(n^{2}\right)$ for a given subgraph $F$.

In view of Theorem 4.8 the following statement seems to be true. 
Conjecture 4.10 There is a polynomial algorithm for the Hamiltonian cycle problem in SMDs.

Theorem 4.8 provides a short proof of Lemma 3.2 and hence of Theorem 3.1, Theorems $5.3,5.7$ as well as of the following result originally obtained in [43].

Theorem 4.11 Let $p(D)$ be the number of vertices in a maximum 1-diregular subgraph of $D, t(D)$ the minimum number of cycles in a 1-diregular subgraph of $D$ with $p(D)$ vertices. Then

1) If $D$ is a strongly connected $S M D$ then the length of a longest cycle in $D$ is at least $p(D)-t(D)+1$.

2) For every integers $c \geq 2, t \geq 1$, there exists a strongly connected $S M D D$ having a maximum 1-diregular spanning subgraph $F$ with $t(D)=t$ cycles each of them of length c such that a longest cycle of $D$ has exactly $p(D)-t(D)+1=t(c-1)+1$ vertices.

3) Suppose $D$ is a strongly connected $S M D$. Given a maximum 1-diregular subgraph in $D$ having $t^{\prime}$ cycles (such a subgraph can be constructed in time $O\left(n^{3}\right)$ ), a cycle with length at least $p(D)-t^{\prime}+1$ in $D$ can be found in time $O\left(n^{2}\right)$.

The second part of Theorem 4.11 can be proved using the following $m$-partite $(m \geq 3)$ tournament $G(c, t), c \geq 2, t \geq 1$ with partite sets $W_{1}, \ldots, W_{m} . G(c, t)$ contains the 1-difactor: $C_{1} \cup C_{2} \cup \cdots \cup C_{t}$, where $C_{i}=\left(x_{1}^{i}, x_{2}^{i}, \ldots, x_{c}^{i}, x_{1}^{i}\right), i=1,2, \ldots, t$. In addition, for each $i=1, \ldots, t$ the vertices $x_{2}^{i}, x_{c}^{i}$ are contained in $W_{3}$, and if $i$ is even then $x_{1}^{i} \in W_{2}$, otherwise $x_{1}^{i} \in W_{1}$. For each $i=1, \ldots, t, A\left(C_{i}\right) \subset\left\{\left(x_{k}^{i}, x_{s}^{i}\right): 1 \leq k<s \leq c\right\} \cup\left\{\left(x_{c}^{i}, x_{1}^{i}\right)\right\} \backslash\left\{\left(x_{1}^{i}, x_{c}^{i}\right)\right\}$. For every $1 \leq i+1<j \leq t$, all arcs between $C_{i}$ and $C_{j}$ are oriented from $C_{i}$ to $C_{j}$ and for every $i=1, \ldots, t-1$, all arcs between $C_{i}$ and $C_{i+1}$ are oriented from $C_{i}$ to $C_{i+1}$ except the arc $\left(x_{1}^{i}, x_{1}^{i+1}\right)$ which has the opposite direction. It is easy to see that the longest cycle of $G(c, t)$ has exactly $t(c-1)+1$ vertices.

Theorem 4.11 implies immediately the following result by J. Ayel (cf. [49]).

Corollary 4.12 If $C$ is a longest cycle of a strongly connected $S M D D$ then $D-C$ has no cycles.

One of the interesting classes of MT is the set of diregular $k$-partite tournaments $(k \geq 2)$. Theorem 5.3 implies that every diregular bipartite tournament is Hamiltonian. This result was first obtained in [49], [78]. Moreover, C.-Q. Zhang [79, 80] proved the following:

Theorem 4.13 There is a cycle of length at least $n-1$ in any diregular MT of order $n$.

C.-Q. Zhang [80] formulated the following conjecture.

Conjecture 4.14 Every diregular MT is Hamiltonian.

Obviously, the number of vertices $s$ in each partite set of a diregular MT is the same. Using Theorem 4.13, the author has verified the last conjecture for $s=2$. 


\section{Cycles in SBDs and ordinary SMDs}

In the survey by L. W. Beineke [18], the following sufficient conditions for a bipartite tournament to have a cycle of length at least $2 r$ due to B. Jackson [49] are given:

Theorem 5.1 Let $T$ be a strongly connected BT with the property that for all vertices $v$ and $w$, either $(v, w) \in A(T)$ or $d^{+}(v)+d^{-}(w) \geq r$. Then $T$ has a cycle of length at least $2 r$.

This supplies a sufficient condition for a BT to be Hamiltonian by taking $r=n / 2$. J. Z Wang $[75,76]$ showed the following result improving Theorem 5.1 in the Hamiltonian case.

Theorem 5.2 Let $T$ be a strongly connected BT with $m$ vertices in each partite set. If for any pair vertices $v$ and $w$ of $T,(v, w) \in A(T)$ implies $d^{+}(v)+d^{-}(w) \geq m-1$, then $T$ is Hamiltonian, unless $T$ is isomorphic to $B\left(\frac{m+1}{2}, \frac{m+1}{2}, \frac{m-1}{2}, \frac{m-1}{2}\right)$ when $m$ is odd or $B\left(\frac{m}{2}, \frac{m-2}{2}, \frac{m}{2} \frac{m+2}{2}\right)$ when $m$ is even.

The following necessary and sufficient conditions for the existence of a Hamiltonian cycle in a semicomplete bipartite digraph have appeared in [30, 31, 46, 53, 55].

Theorem 5.3 A semicomplete bipartite digraph $D$ is Hamiltonian, if and only if $D$ is strongly connected and has a 1-diregular spanning subgraph. There exists an $O\left(n^{2.5} / \sqrt{\log n}\right)$ algorithm for finding a Hamiltonian cycle in a SBD D (if D is Hamiltonian).

The complexity of the algorithm mentioned in Theorem 5.3 is dominated by that of the algorithm [3] for finding a maximum matching in a bipartite graph. The proofs of Theorem 5.3 in $[30,31,55]$ are based on the following:

Lemma 5.4 If a strongly connected $S B D D$ has a 1-difactor $F=C \cup Z$ containing two cycles, then $D$ is Hamiltonian. Given $F$ one can find a Hamiltonian cycle in $D$ in time $O(|V(C)||V(Z)|)$.

Lemma 5.4 can be proved in a rather simple way using Theorem 4.8 [11]. An algorithm for checking whether a SBD $D$ contains a Hamiltonian cycle and finding one if $D$ is Hamiltonian consists of the following steps.

1) Check whether $D$ is strongly connected applying any $O\left(n^{2}\right)$-time algorithm (e. g. the one in [70]). If $D$ is not strongly connected, then $D$ is not Hamiltonian.

2) Find in $D$ a maximum 1-diregular subgraph $F$ (apply the construction described before Algorithm 3.3). If $F$ is not a 1-difactor of $D$, then $D$ is not Hamiltonian.

3) Construct a semicomplete digraph $T=T(F)$ as follows. The vertices of $T$ are the cycles of $F$. A cycle $C_{1}$ of $F$ dominates another cycle $C_{2}$ in $T$ if and only if there is an arc in $D$ from $C_{1}$ to $C_{2}$. Find a Hamiltonian cycle $H$ in $T(F)$ using the algorithm from [54].

4) Transform $H$ into a Hamiltonian cycle of $D$ using Lemma 5.4.

J. Bang-Jensen proved [10] that Theorem 5.3 remains true for arc-local tournament digraphs. Recently, J. Bang-Jensen, M. El Haddad, Y. Manoussakis and T. Przytycka [13] 
obtained a random parallel algorithm for checking whether a SBD $D$ has a Hamiltonian cycle and finding one (if there is) in time $O\left(\log ^{4} n\right)$ using a CRCW PRAM with $O\left(n^{2}\right)$ processors (see, e. g., [50] for the definition of CRCW PRAM).

It follows from Theorem 4.11 that the first part of Theorem 5.3 cannot be extended to the entire set of semicomplete $t$-partite digraphs $(t \geq 3)$.

M. Manoussakis and Y. Manoussakis [52] determined the number of non-isomorphic BTs with $2 m$ vertices containing a unique Hamiltonian cycle. Let $h_{m}$ be the number of such BTs. It is shown in [52] that $h_{2}=h_{3}=1$ and $h_{m}=4 h_{m-1}+h_{m-2}$ for $m \geq 4$. R. J. Douglas [45] gave a structural characterization of tournaments having a unique Hamiltonian cycle. This result implies a formula for the number $s_{n}$ of non-isomorphic tournaments of order $n$ with a unique Hamiltonian cycle. This characterization as well as formula are rather complicated. M. R. Garey [26] later showed that $s_{n}$ could be expressed as a Fibonacci number $\left(s_{n}=f_{2 n-6}\right)$; his derivation was based on Douglas's characterization. J. W. Moon [57] obtained a direct proof of Garey's formula that is essentially independent of Douglas's characterization.

We make the following trivial but useful observation. The length of a longest cycle in any digraph is equal to the maximum length of a longest cycle in its strongly connected components. Hence, solving the longest cycle problem, one may consider only strongly connected digraphs. In [38] the following result which gives a complete solution of the longest cycle problem in the case SBDs was obtained.

Theorem 5.5 1) The length of a longest cycle in a strongly connected $S B D D$ is equal to the number of vertices of a 1-diregular subgraph of $D$ of maximum cardinality. 2) There exists an algorithm for finding a longest cycle in a strongly connected $S B D D$ in time $O\left(n^{3}\right)$.

It easy to see that Theorem 5.5 follows from Lemmas 4.9, 5.4 and the construction described before Algorithm 3.3. The algorithm mentioned in Theorem 5.5 is just a modification of that described after Lemma 5.4.

Theorem 5.3 as well as Theorem 5.5 can be proved for the class of ordinary semicomplete $t$-partite digraphs $(t \geq 3)$ with a little alteration. Indeed, the following two claims hold [38].

Theorem 5.6 1) Let $D$ be a strongly connected ordinary SMD. Then for any 1-diregular subgraph $F$ of maximum cardinality of $D$, there is a cycle $C$ of $D$ satisfying $V(C)=V(F)$ (Clearly each such cycle is a longest cycle of $D)$. 2) There exists an $O\left(n^{3}\right)$ algorithm for constructing a longest cycle in any strongly connected ordinary SMD D.

Theorem 5.7 An ordinary SMD D is Hamiltonian if and only if D is strongly connected and has a 1-diregular spanning subgraph. Finding a Hamiltonian cycle in a Hamiltonian ordinary SMD D can be done in time $O\left(n^{2.5} / \sqrt{\log n}\right)$.

Let $T=T\left(x_{1}, \ldots, x_{n}\right)$ be a semicomplete digraph with $V(T)=\left\{x_{1}, \ldots, x_{n}\right\}$, and let $k_{i}$ be non-negative integers $(1 \leq i \leq n)$. A closed $\left(k_{1}, k_{2}, \ldots, k_{n}\right)$-walk of $T$ is a closed directed walk of $T$ visiting each vertex $x_{j}$ no more than $k_{j}$ times (the first and the last vertices of a closed walk coincide and are considered as a single vertex). 
Let

$$
D_{T}=D_{T}\left(x_{1}^{k_{1}}, x_{2}^{k_{2}}, \ldots, x_{n}^{k_{n}}\right)
$$

denote the ordinary SMD with partite sets $V_{i}\left(\left|V_{i}\right|=k_{i}\right.$ and if $k_{i}=0$, then $V_{i}=\emptyset$, i.e. $V_{i}$ is absent in $T), 1 \leq i \leq n$, and

$$
A\left(D_{T}\right)=\bigcup\left(V_{i} \times V_{j}: k_{i} k_{j} \neq 0, \quad\left(x_{i}, x_{j}\right) \in A(T), \quad 1 \leq i \neq j \leq n\right) .
$$

Theorem 5.6 implies

Corollary 5.8 The maximum length of a closed $\left(k_{1}, k_{2}, \ldots, k_{n}\right)$-walk of a strongly connected semicomplete digraph $T\left(x_{1}, x_{2}, \ldots, x_{n}\right)$ is equal to the number of vertices of a maximum 1diregular subgraph of the digraph $D_{T}\left(x_{1}^{k_{1}}, x_{2}^{k_{2}}, \ldots, x_{n}^{k_{n}}\right)$.

L. Moser [47] and J. Moon [58] strengthened the theorem of Camion mentioned above and proved, respectively, that a strongly connected tournament is pancyclic and, even, vertex pancyclic.

The following characterizations of even pancyclic and vertex even pancyclic bipartite tournaments were derived in [19] and [77], respectively. Note, that the last characterization was obtained independently in [46] as well.

Theorem 5.9 A bipartite tournament is even pancyclic as well as vertex even pancyclic if and only if it is Hamiltonian and is not isomorphic to the bipartite tournament $B(r, r, r, r)(r=$ $2,3, \ldots)$.

Considering diregular bipartite tournaments, D. Amar and Y. Manoussakis [5] and, independently, J. Z. Wang [74] showed the following:

Theorem 5.10 An $r$-diregular BT is arc even pancyclic unless it is isomorphic to $B(r, r, r, r)$.

The analogous result for diregular tournaments was obtained by B. Alspach in [4] (every diregular tournament is arc pancyclic). Z. M. Song [67] studied complementary cycles in BTs which are similar to diregular ones. He proved the following:

Theorem 5.11 Let $R$ be a BT with $2 k+1$ vertices in each partite set $(k \geq 4)$. If every vertex of $R$ has outdegree and indegree at least $k$ then for any vertex $x$ in $R, R$ contains a pair of disjoint cycles $C, Q$ such that $C$ includes $x$ and the length of $C$ is at most 6 unless $R$ is isomorphic to $B(k+1, k+1, k, k)$.

Observe that a characterization of even pancyclic (and vertex even pancyclic) semicomplete bipartite digraphs coincides with the above-mentioned one. Indeed, the result follows from the fact that any bipartite tournament obtained by the reorientation of an arc of $B(r, r, r, r)$ is Hamiltonian, and so, vertex even pancyclic. Combining these results with the above described necessary and sufficient conditions for the existence of a Hamiltonian cycle in a 
semicomplete bipartite digraph (Theorem 5.3) we obtain a polynomial characterization for the above properties.

A characterization of pancyclic (and vertex pancyclic) ordinary $m$-partite $(m \geq 3)$ tournaments was established in [36]. As opposed to the characterization of even pancyclic semicomplete bipartite graphs the last one does not imply immediately a characterization of pancyclic (or vertex pancyclic) ordinary semicomplete $m$-partite digraphs. Indeed, there exist vertex pancyclic ordinary SMDs which contain no Hamiltonian ordinary multipartite tournaments as spanning subgraphs. Such examples are semicomplete $m$-partite digraphs $S_{m, r}$ with $r$ vertices in each partite set but one and $(m-1) r$ vertices in the last one $(r \geq 1, m \geq 3)$. A semicomplete $m$-partite digraph is called a complete $m$-partite digraph if it has the arcs $(u, v),(v, u)$ for any pair $u, v$ in distinct partite sets. $S_{m, r}$ is vertex pancyclic by Theorem 5.12 (see below) and it has no Hamiltonian ordinary $m$-partite tournament as a spanning subgraph since any Hamiltonian cycle of $S_{m, r}$ must alternate between the largest partite set and the other partite sets and hence it cannot be a subgraph of an ordinary multipartite tournament.

An ordinary SMD $D$ is called a zigzag digraph if it has more than four vertices and $k(\geq 3)$ partite sets $V_{1}, V_{2}, V_{3}, \ldots, V_{k}$ such that $A\left(V_{2}, V_{1}\right)=A\left(V_{i}, V_{2}\right)=A\left(V_{1}, V_{i}\right)=\emptyset$ for any $i \in\{3,4, \ldots, k\},\left|V_{1}\right|=\left|V_{2}\right|=\left|V_{3}\right|+\left|V_{4}\right|+\cdots+\left|V_{k}\right|$.

Observe that any cycle in such a graph has the same number, say $s$, of vertices from $V_{1}$ and $V_{2}$ and at least $s$ vertices from $V_{3} \cup \cdots \cup V_{k}$. Therefore, $H$ has no prehamiltonian cycle, i.e. a cycle containing all vertices of $H$ but one. Observe also that an ordinary 4-partite tournament with more than four vertices is not a pancyclic digraph. Indeed, the single (up to isomorphism) strongly connected tournament with four vertices has no closed directed walk of length five.

The following characterization of pancyclic and vertex pancyclic ordinary SBD was obtained in [42].

Theorem 5.12 1) An ordinary semicomplete $k$-partite digraph $(k \geq 3) D$ is pancyclic if and only if

i) $D$ is strongly connected;

ii) it has a 1-diregular spanning subgraph;

iii) it is neither a zigzag digraph nor a 4-partite tournament with at least five vertices.

2) A pancyclic ordinary semicomplete $k$-partite digraph $D$ is vertex pancyclic if and only if either

i) $k>3$ or

ii) $k=3$ and $D$ contains two 2 -cycles $Z_{1}, Z_{2}$ such that $Z_{1} \cup Z_{2}$ has vertices in three partite sets. 
3) There exists an $O\left(n^{2.5} / \sqrt{\log n}\right)$ algorithm for determining whether an ordinary semicomplete $k$-partite $(k \geq 3)$ digraph $D$ is pancyclic (vertex pancyclic).

The following result conjectured in [46] was proved in [15].

Theorem 5.13 1) If $D$ is a k-strongly connected bipartite tournament, $k \geq 1$, then $D$ is $k$-vertex cyclic. 2) There exists a $O\left(n^{3}\right)$ algorithm to find a cycle through any set of $k$ vertices in a k-strongly connected bipartite tournament.

The $(k-1)$-strongly connected BT $B=B(p-k, q-k+1, k, k-1)(p>q \geq 2 k-2 \geq 2)$ considered in [46] shows that Theorem 5.13 is best possible in terms of connectivity ( $B$ is non- $k$-vertex cyclic). J. Bang-Jensen and Y. Manoussakis [15] raised the following conjecture.

Conjecture 5.14 For every fixed $k$ there exists a polynomial algorithm to decide the existence of a cycle through a given set of $k$ vertices in a BT and to find one if it exists.

Y. Manoussakis and Z. Tuza [55] have already proved this conjecture for $k=2$.

The situation with $k$-cyclic ordinary SMD is better. J. Bang-Jensen, G. Gutin and J. Huang derived in [11] a complete characterization of $k$-cyclic ordinary SMDs. They showed the following:

Theorem 5.15 An ordinary SMD D is k-cyclic if and only if for every set $Z$ of $k$ vertices, there exists a 1-diregular subgraph of $D$ containing all the vertices of $Z$. There exists an $O\left(n^{2.5} / \sqrt{\log n}\right)$ algorithm to decide if there is a cycle meeting a given set $Z$ of $k$ vertices and find one if it exists.

J. Bang-Jensen and Y. Manoussakis [15] also described arc variants of Theorem 5.13 and Conjecture 5.14.

Theorem 5.16 There exists a function $f(k)$ such that any $f(k)$-strongly connected BT is $k$-arc cyclic.

Conjecture 5.17 For any fixed natural number $k$ there exists a polynomial algorithm to decide the existence of a cycle through $k$ given arcs in a BT and to find one if it exists.

B. Jackson [49] suggests that Kelly's conjecture remains valid for diregular BTs, i. e. he raises the following

Conjecture 5.18 Every diregular BT is decomposable into Hamiltonian cycles.

In support for this conjecture, D. Amar and Y. Manoussakis [5] proved the following:

Theorem 5.19 Let $D$ be an r-diregular bipartite tournament with partite sets $X$ and $Y$. Colour all the arcs from $X$ to $Y$ red. Then $D$ contains exactly $r$ Hamiltonian cycles no two of which share a red edge. 


\section{Cycles and paths in semicomplete digraphs}

What is the complexity of the Hamiltonian path and cycle problems in tournaments? The inductive classical proof of Redei's theorem gives at once a simple $O\left(n^{2}\right)$ algorithm for the first problem. Since sorting corresponds to finding a Hamiltonian path in a transitive tournament, we have an $O(n \log n)$-time algorithm in this case. P. Hell and M. Rosenfeld [48] obtained an algorithm with the same complexity solving the Hamiltonian path problem for any tournament. The well known proof of Moon's theorem provides an $O\left(n^{3}\right)$-time algorithm for the Hamiltonian cycle problem. Y. Manoussakis [54] constructed an $O\left(n^{2}\right)$-time algorithm for this problem.

A parallel algorithm $A$ for a problem with size $n$ is called an $N C$-algorithm if there are constants $k, l$ such that $A$ can be performed in time $O\left(\log ^{k} n\right)$ on an $O\left(n^{l}\right)$ processors PRAM. We refer the reader to [50] for a discussion of $N C$-algorithms. D. Soroker [68] studies the parallel complexity of the above mentioned problems. He proved the following:

Theorem 6.1 There are NC-algorithms for the Hamiltonian path and Hamiltonian cycle problems in tournaments.

Another $N C$-algorithm for the Hamiltonian path problem in tournaments has been obtained by J. Naor [59]. As to the Hamiltonian path problem for tournaments, the most effective parallel algorithm is due to A. Bar-Noy and J. Naor [7]. They constructed an algorithm performed in time $O(\log n)$ on an $O(n)$ processors CRCW PRAM for a tournament containing $n$ vertices. The most effective parallel algorithm for the Hamiltonian cycle problem for tournaments is due to E. Bampis, M. El Haddad, Y. Manoussakis and M. Santha [6]. They found a fast parallel procedure which transforms the Hamiltonian cycle problem into the Hamiltonian path one in the following sense: Given a Hamiltonian path in a tournament as input, the procedure constructs a Hamiltonian cycle. The parallel running time of the procedure is $O(\log n)$ using $O\left(n^{2} / \log n\right)$ processors in the CRCW model.

J. Bang-Jensen, Y. Manoussakis and C. Thomassen [16] obtained a polynomial algorithm solving the problem (which appears in $[60,68]$ ) of deciding the existence of a Hamiltonian path with prescribed initial and terminal vertices in a tournament. Obviously, the last problem is equivalent to the problem of existence of a Hamiltonian cycle containing a given arc in a tournament. They raised the following:

Conjecture 6.2 For each fixed $k$, there exists a polynomial algorithm for deciding if there exists a Hamiltonian cycle through $k$ prescribed arcs in a tournament.

The $k$-arc cyclic problem is the following: Given $k$ distinct arcs in a digraph $D$, decide whether $D$ has a cycle through all the arcs. Bang-Jensen and Thomassen [17] considered this problem for semicomplete digraphs. They proved:

Theorem 6.3 There exists a polynomial algorithm for deciding if two independent arcs lie on a common cycle in a semicomplete digraph. 
They also showed that if $k$ is part of the input then the above problem is $N P$-complete.

Theorem 6.4 The $k$-arc cyclic problem is NP-complete even for semicomplete and semicomplete bipartite digraphs.

Sufficient conditions for semicomplete digraphs and tournaments to be 2-arc cyclic are studied in [9] where the following theorem is proved.

Theorem 6.5 Every 5-strongly connected semicomplete digraph is 2-arc cyclic; every 3connected tournament is 2-arc cyclic.

In [9] it is noted that both results are best possible in terms of the required connectivity.

A digraph $D$ is said to be transitive if $(x, y),(y, z) \in A(D)$ implies $(x, z) \in A(D)$. This notion has been generalized by F. Harary (cf. [73]) as follows: $D$ is $(m, k)$-transitive $(m>k \geq 1)$ if for every path $P$ of length $m$ there exists a path $Q$ of length $k$ with the same endvertices, such that $V(Q) \subset V(P)$. A. Gyárfás, M.S. Jacobson and L.F. Kinch [44] studied $(m, k)$-transitivity and obtained a characterization of $(m, 1)$-transitive tournaments for $m \geq 2$. Using another approach, Z. Tuza [73] characterized $(m, 1)$-transitive semicomplete digraphs for every $m \geq 2$ and $k=1$.

Theorem 6.6 Let $D$ be a semicomplete digraph, and let $m$ be an integer, $m \geq 2$. Then the following statements are equivalent.

(a) $D$ is $(m, 1)$-transitive.

(b) No cycle of length greater than $m$ contains any arc $(x, y)$ of $D$ such that $(y, x) \notin A(D)$.

(c) Every strong component of $D$ with more than $m$ vertices induces a semicomplete digraph.

Obviously, this characterization provides an $O\left(n^{2}\right)$-time algorithm for finding the minimum $m$ such that a given semicomplete digraph $D$ is $(m, 1)$-transitive. Z. Tuza [73] also obtained a characterization of $(3,2)$-transitive semicomplete digraphs.

\section{The number of paths and cycles in MTs}

The main problems in the topic of this section are the following:

1) Find the maximum possible number of $s$-cycles (s-paths) in a MT with a given number of vertices in each partite set.

2) Find the minimum possible number of $s$-cycles in a strongly connected MT with a given number of vertices in each partite set.

These problems were completely solved only in some special cases. The first problem (on cycles) was solved for tournaments when $s=3,4$ and for BTs when $m=4$ (cf. [18]).

Solving an old conjecture of T. Szele [69], N. Alon [2] showed: 
Theorem 7.1 The maximum number, $P(n)$, of Hamiltonian paths in a tournament on $n$ vertices satisfies

$$
P(n) \leq c n^{1.5} n ! / 2^{n-1}
$$

where $c$ is independent of $n$.

The short proof of Theorem 7.1 is based on Minc's Conjecture [56] on permanents of (0,1)-matrices proved by Bregman [24].

Szele [69] proved that

$$
P(n) \geq n ! / 2^{n-1}
$$

and hence the gap between the upper and lower bounds for $P(n)$ is only $O\left(n^{1.5}\right)$.

It would be interesting to close this gap and determine $P(n)$ up to a constant factor.

The second problem was completely solved for tournaments for all $s$ and for BTs when $s=4$ (cf. [18]). For MTs the following two results [28] were obtained.

Theorem 7.2 Let $T$ be a strongly connected m-partite tournament, $m \geq 3$. Then $T$ contains at least $m-2$ 3-cycles.

Theorem 7.3 Let $G$ be a complete m-partite graph, $m \geq 3$, which is not isomorphic to $K_{2,2, \ldots, 2}$ for odd $m$. Then there exists a strong orientation of $G$ with exactly $m-2$ 3-cycles.

\section{Acknowledgment}

I would like to thank N. Alon for fruitful comments, J. Bang-Jensen, P. Camion, P. Hell, J. Huang, Y. Manoussakis, C.-Q. Zhang and Z. Tuza for helpful information, and the anonymous referees for a number of important suggestions.

\section{References}

[1] Aigner M., Combinatorial Theory. Springer-Verlag, Berlin, 1979.

[2] Alon N., The maximum number of hamiltonian paths in tournaments. Combinatorica 10 (1990) 319-324.

[3] Alt H., Blum N., Melhorn K., and Paul M., Computing of maximum cardinality matching in a bipartite graph in time $O\left(n^{1.5} \sqrt{m / \log n}\right)$. Inf. Proc. Letters 37 (1991) 237-240.

[4] Alspach B., Cycles of each length in regular tournaments. Canad. Math. Bull. 10 (1967) 283-285.

[5] Amar D., Manoussakis Y., Cycles and paths of many lengths in bipartite digraphs. J. Comb. Theory Ser. B 50 (1990) 254-264. 
[6] Bampis E., El Haddad E., Manoussakis Y., and Santha M., A parallel reduction of hamilton cycle to hamilton path in tournaments (submitted).

[7] Bar-Noy A., Naor J., Sorting, minimal feedback sets, and hamiltonian paths in tournaments. SIAM J. Disc. Math. 3 (1990) 7-20.

[8] Balakrishnan R., Paulraja P., Note on the existence of directed $(k+1)$-cycle in diconnected complete $k$-partite digraphs. J. Graph Theory 8 (1984) 423-426.

[9] Bang-Jensen J., On the 2-linkage problem for semicomplete digraphs. Annals of Disc. Math. 41 (1989) 23-38.

[10] Bang-Jensen J., Arc-local tournament digraphs: a generalization of tournaments and bipartite tournaments, Institut for Matematik og Datalogi, Odense Univ., Preprint, No. 2, 1993.

[11] Bang-Jensen J., Gutin G., Huang J., A sufficient condition for a complete multipartite digraph to be Hamiltonian (submitted).

[12] Bang-Jensen J., Gutin G., Huang J., Weekly Hamiltonian-connected ordinary multipartite tournaments (submitted).

[13] Bang-Jensen J., Haddad M. E., Manoussakis Y., Przytycka T., Parallel algorithms for the Hamiltonian cycle and Hamiltonian path problems in semicomplete bipartite digraphs (submitted).

[14] Bang-Jensen J., Manoussakis Y., Weakly hamiltonian-connected vertices in bipartite tournaments (submitted).

[15] Bang-Jensen J., Manoussakis Y., Cycles through $k$ vertices and $k$ arcs in bipartite tournaments. Institut for Matematik og Datalogi, Odense Univ., Preprint, 1991, No. 9.

[16] Bang-Jensen J., Manoussakis Y., Thomassen C., A polynomial algorithm for Hamiltonian-connectedness in semicomplete digraphs. J. Algorithms 13 (1992) 114-127.

[17] Bang-Jensen J., Thomassen C., A polynomial algorithm for the 2-path problem for semicomplete digraphs. SIAM J. Discrete Math. 5 (1992) 366-376.

[18] Beineke L.W., A tour through tournaments or bipartite and ordinary tournaments: a comparative survey. J. London Math. Soc. Lect. Notes Ser. No. 52 (1981) 41-55.

[19] Beineke L.W., Little C., Cycles in bipartite tournaments. J. Combin. Theory Ser. B. 32 (1982) 140-145.

[20] Beineke L.W., Wilson R.J., A survey of recent results on tournaments. Recent Adv. Graph Theory. Proc. Symp., Prague, 1974. Academia, Praha (1975) 31-48. 
[21] Bermond J.-C., Thomassen C., Cycles in digraphs: a survey. Preprint Mat. Inst. Aarhus Univ., No. 10 (1980/1981).

[22] Bondy J.A., Diconnected orientation and a conjecture of Las Vergnas J. London Math. Soc. 14 (1976) 277-282.

[23] Bondy J. A., Murty U. R. S. Graph Theory with Applications, MacMillan Press, 1976.

[24] Bregman L. M., Some properties of nonnegative matrices and their permanents. Soviet Math. Dokl. 14 (1973) 945-949.

[25] Camion P., Chemie et circuits hamiltoniens des graphes complets. C.R. Acad. Sci. Paris 249 (1959) 2151-2152.

[26] Garey M. R., On enumerating tournaments that admit exactly one Hamiltonian circuit. J. Combin. Theory Ser. B 13 (1972) 266-269.

[27] Goddard W., Kubicki G., Oellermann O., Tian S., On multipartite tournaments. J. Comb. Theory Ser. B 47 (1991) 284-300.

[28] Goddard W., Oellermann O., On the cycle structure of multipartite tournaments. Graph Theory, Combinatorics and Applications. Wiley-Interscience 1 (1991) 525-533.

[29] Guo Y., Volkmann L., Cycles in multipartite tournaments (submitted).

[30] Gutin G., On cycles in complete n-partite digraphs. Gomel Polytechnic Inst. 1982, Depon. in VINITI 17.05.82, No. 2473 (In Russian).

[31] Gutin G., A criterion for complete bipartite digraphs to be Hamiltonian. Vestsi Acad. Navuk BSSR Ser. Fiz.-Mat. Navuk No. 1 (1984) 99-100 (In Russian).

[32] Gutin G., On cycles in strong n-partite tournaments. Vestsi Acad. Navuk BSSR Ser. Fiz.-Mat. Navuk No. 5 (1984) 105-106 (In Russian).

[33] Gutin G., Effective characterization of complete bipartite digraphs that have a Hamiltonian path. Kibernetica No. 4 (1985) 124-125 (In Russian).

[34] Gutin G., The radii of n-partite tournaments. Math. Notes 40 (1986) 743-744.

[35] Gutin G., A characterization of complete $n$-partite digraphs that have a Hamiltonian path. Kibernetica No. 1 (1988) 107-108 (In Russian).

[36] Gutin G., A characterization of vertex pancyclic partly oriented $k$-partite tournaments. Vestsi Acad. Navuk BSSR, Ser. Fiz.-Mat. Navuk. No. 2 (1989) 41-46 (in Russian). 
[37] Gutin G., m-sources in complete multipartite digraphs. Vestsi Acad. Navuk BSSR Ser. Fiz.-Mat. Navuk No. 5 (1989) 101-106 (In Russian).

[38] Gutin G., Efficient algorithms for finding the longest cycles in certain complete multipartite digraphs. Technical report No. 256 of the Eskenasy Inst. of Computer Sciences, Tel-Aviv Univ., 1992.

[39] Gutin G., A note on cycles in multipartite tournaments. J. Combinatorial Theory Ser. $B 58$ (1993) 319-321.

[40] Gutin G., Finding a longest path in a complete multipartite digraph. SIAM J. Discrete Math. 6 (1993) 270-273.

[41] Gutin G., Minimizing and maximizing the diameter in orientations of graphs. Graphs and Combin. (to appear).

[42] Gutin G., Characterizations of vertex pancyclic and pancyclic ordinary complete multipartite digraphs. Discrete Math. (to appear).

[43] Gutin G., A note on long cycles in complete multipartite digraph (submitted).

[44] Gyárfás A., Jacobson M. S., Kinch L. F., On a generalization of transitivity for digraphs. Discrete Math. 69 (1988) 35-41.

[45] Douglas R J., Tournaments that admit exactly one Hamiltonian circuit. Proc. London Math. Soc. 21 (1970) 716-730.

[46] Häggkvist R., Manoussakis Y., Cycles and paths in bipartite tournaments with spanning configurations. Combinatorica 9 (1989) 33-38.

[47] Harary F., Moser L., The theory of round robin tournaments. Amer. Math. Monthly 73 (1966) 231-246.

[48] Hell P., Rosenfeld M., The complexity of finding generalized paths in tournaments. $J$. Algorithms 4 (1983) 303-309.

[49] Jackson B., Long paths and cycles in oriented graphs. J. Graph Theory 5 (1981) 145-157.

[50] Karp R.M., Ramachandran V., A survey of parallel algorithms for shared memory machines. Handbook of Theoretical Computer Science, Chapter 17, MIT Press (1990) 871-941.

[51] Landau H.G., On dominance relations and the structure of animal societies, III. Bull. Math. Biophys. 15 (1953) 143-148. 
[52] Manoussakis M., Manoussakis Y., The number of bipartite tournaments with a unique given factor. J. Graph Theory 13 (1989) 359-368.

[53] Manoussakis Y., Problemes extremaux dans les graphes orientes. Ph.D. thesis, Universite Paris-Sud, Orsay, 1987.

[54] Manoussakis Y., A linear time algorithm for finding Hamiltonian cycle in tournaments. Discrete Appl. Math. 36 (1992) 199-202.

[55] Manoussakis Y., Tuza Z., Polynomial algorithms for finding cycles and paths in bipartite tournaments. SIAM J. Disc. Math. 3 (1990) 537-543.

[56] Minc H., Upper bounds for permanents of (0,1)-matrices. Bull. Amer. Math. Soc. 69 (1963) 789-791.

[57] Moon J. W., The number of tournaments with a unique spanning cycle. J. Graph Theory 6 (1982) 303-308.

[58] Moon J. W., Topics on Tournaments. Holt, Rinehart and Winston, N.Y., 1968.

[59] Naor J., Two parallel algorithms in graph theory. Technical Report CS-86-6, Hebrew University, 1986.

[60] Ore O., Theory of Graphs. AMS Colloquium Publications 38, AMS, Providence, 1962.

[61] Papadimitriou H., Steiglitz K., Combinatorial Optimization: Algorithms and Complexity. Prentice-Hall Inc., New Jersey, 1982.

[62] Petrovic V., Thomassen C., Kings in k-partite tournaments. Discrete Math. 98 (1991) $237-238$.

[63] Redei L., Ein kombinatoricher Satz. Acta Litt. Szeged 7 (1934) 39-43.

[64] Robbins H.E., Theorem on graphs, with an application to a problem of traffic control. Amer. Math. Month ly 46 (1929) 281-283.

[65] Sands B., Sauer N., Woodrow R., On monochromatic paths in edge-coloured digraphs. J. Combinatorial Theory Ser. B 33 (1982) 271-275.

[66] Soltes L., Orientations of graphs minimizing the radius or the diameter. Math. Slovaca 38 (1986) $289-296$.

[67] Song Z. M., Complementary cycles in bipartite tournaments. J. Nanjing Inst. Tech. 18 (1988) 32-38. 
[68] Soroker D., Fast parallel algorithms for finding Hamiltonian paths and cycles in tournaments. J. Algorithms 9 (1988) 276-286.

[69] Szele T., Kombinatorikai vizsgalatok az iranyitott teljes graffal kapcsolatban. Mat. Fiz. Lapok 50 (1943) 223-256.

[70] Tarjan R. E., Depth-first search and linear graph algorithms. SIAM J. Computing 1 (1972) 146-160.

[71] Thomassen C., Hamiltonian connected tournaments. J. Comb. Theory 28 (1980) 142163.

[72] Thomassen C., Edge-disjoint hamiltonian paths and cycles in tournaments. Proc. London Math. Soc. 45 (1982) 151-168.

[73] Tuza Z., Characterization of $(m, 1)$-transitive and (3,2)-transitive semi-complete directed graphs. Discrete Math. (to appear)

[74] Wang J. Z., Cycles of all possible lengths in diregular bipartite tournaments. Ars Combinatoria 32 (1991) 279-288.

[75] Wang J. Z., Long cycles in bipartite tournaments. Discrete Math. (to appear).

[76] Wang J. Z., A sufficient condition for hamiltonian cycles in bipartite tournaments. Australasian J. of Comb. 5 (1992) 299-304.

[77] Zhang K.-M., Vertex even-pancyclicity in bipartite tournaments. J. Nanjing Univ. Math. Biquarterly 1 (1981) 85-88.

[78] Zhang C.-Q., The longest paths and cycles in bipartite oriented graphs. J. Mathematical Research and Exposition 1 (1981) 35-38.

[79] Zhang C.-Q. Some results on tournaments. J. Qufu Teachers Colledge No. 1 (1985) 51-53.

[80] Zhang C.-Q., Hamilton paths in multipartite oriented graphs. Annals of Disc. Math. 41 (1989) 499-514. 